\title{
Linx
}

Revue des linguistes de l'université Paris X Nanterre

$60 \mid 2009$

Nonne scripta manent?

\section{Le voyage à Pau avec Jacques Anis : retour sur le colloque de 1997 "Propriétés de l'écriture »}

Jean-Louis Chiss et Christian Puech

\section{(2) OpenEdition}

\section{Journals}

Édition électronique

URL : http://journals.openedition.org/linx/695

DOI : $10.4000 /$ linx.695

ISSN : 2118-9692

Éditeur

Presses universitaires de Paris Nanterre

\section{Édition imprimée}

Date de publication : 1 mai 2009

Pagination : 67-72

ISSN : 0246-8743

\section{Référence électronique}

Jean-Louis Chiss et Christian Puech, «Le voyage à Pau avec Jacques Anis : retour sur le colloque de 1997 «Propriétés de l'écriture » », Linx [En ligne], 60 | 2009, mis en ligne le 03 octobre 2012, consulté le 31 mai 2019. URL : http://journals.openedition.org/linx/695 ; DOI : 10.4000/linx.695 


\title{
Le voyage à Pau avec Jacques Anis : retour sur le colloque de 1997 "Propriétés de l'écriture "
}

\author{
Jean-Louis Chiss et Christian Puech \\ Université Paris 3 - Sorbonne Nouvelle
}

C'était le 12 novembre 1997. Habitués aux petits trajets entre le domicile de Jacques et quelques restaurants d'Asnières, nous avons entrepris, cette fois-là, tous les trois, un grand voyage vers Pau pour participer à un colloque d'importance sur l'écriture, de dimension interdisciplinaire et internationale, organisé par Jean-Gérard Lapacherie. La disparition brutale de Nina Catach, quelques jours avant ce colloque, avait privé l'auditoire d'une figure éminente et interrompu le dialogue entre les positions «autonomistes» dont J. Anis s'était fait le héraut et l'ancrage "phonographiste » que revendiquait N. Catach. Dix ans plus tôt, la journée d'études organisée par N. Catach (Pour une théorie de la langue écrite, 1988) avait donné lieu à une belle confrontation dans laquelle les aspects historiques et épistémologiques avaient trouvé leur place. C'était d'ailleurs le sens de notre contribution au volume de J. Anis (1988) dans un trajet inscrit en histoire des théories du langage où se pose la question de la constitution des objets de connaissance, de leurs émergences et de leurs distributions entre les disciplines ${ }^{1}$. De ce point de vue, l'« écriture », dans son flou terminologique et

\footnotetext{
${ }^{1}$ La prise en compte des évolutions théoriques et des modèles de description, du « pluri-système » de N. Catach et Jean-Pierre Jaffré à l'« autonomie relative» de J. Anis et à l'approfondissement de son travail sur la construction du concept de "graphème ", la (re)découverte de textes importants de linguistes européens et américains sur l'écriture, la transformation des débats sur l'apprentissage du lire-écrire, constituent les éléments essentiels qui nous avaient amenés tous trois à entreprendre une refonte totale de l'ouvrage de 1988. Nous avons tardé, le temps a passé...
} 
la diversité de ses acceptions, figurait un lieu idéal pour les interrogations sur les frontières disciplinaires, outre son évident intérêt en termes de positivités descriptives et d'enjeu pour le champ social et scolaire. C'est dire que, lors de ce colloque de Pau, nombreux furent les prismes théoriques, de Saussure à la linguistique structurale, fonctionnelle et distributionnelle, de la sémiolinguistique à la sémiotique de l'image, de la psychologie cognitive aux études génétiques, avec, cerise sur le gâteau, cette notion de "literacy», déjà familière au monde anglo-saxon et qu'avaient commencé à s'approprier, depuis une dizaine d'années, un certain nombre de chercheurs francophones (du Québec, avec la francisation « littératie », à la France).

\section{Les positions en présence à Pau}

C'est ainsi que, du traitement ambivalent de l'écriture dans le Cours de linguistique générale à sa reprise dans les filiations saussuriennes de la linguistique structurale, le parcours théorique montrait bien, à notre sens, que le spectre des positions des linguistes vis-à-vis du signifiant graphique était très diversifié et souvent inattendu. C'est que, de l'intérieur même du domaine linguistique, le problème de la place d'une théorisation de l'écriture s'était posé en fait très tôt, et ce au cœur même de la discipline, c'est-à-dire dans les débats sur la nature du signe linguistique, les positions relatives de la linguistique et de la sémiologie, où se décidaient à la fois le sort réservé au signifiant graphique et les relations de la linguistique aux disciplines voisines. Avec «l'objectivation du parler» sur "l'écran » des «systèmes d'écriture », c'est toute la réflexion sur la notion de système qui se complexifie : comment articuler les deux acceptions du terme « système » qui renvoient l'une à l'autre quand on parle de système d'écriture et de système de la langue, alors qu'aucune des deux n'est totalement homothétique, ni bien sûr étrangère à l'autre².

Poser que l'écriture représente la langue ne reste une évidence première qu'aussi longtemps qu'on laisse de côté la question de savoir ce que, dans ce cas, signifie « représenter » (reproduire, exprimer, déléguer, attester, transposer, trahir, manifester, enregistrer, transcrire..., etc.). En simplifiant, on peut soutenir que le traitement de la question de l'écriture dans la filiation structuraliste (pragoise, danoise, américaine...) de Saussure n'aura fait que moduler dans des théorisations riches d'effets et de questions ouvertes la note dominante de la « représentation ». Mais ce rappel historique trop rapide n'avait pas d'autre but que d'introduire à une situation à la fois plus contemporaine et plus large.

En effet, l'effort des linguistes pour cartographier l'immense continent-écriture hérite dans une large mesure des cadres conceptuels et des hésitations des pionniers. Or, c'est bien aux limites des principes de la linguistique structurale développée au XX siècle que ramène le signifiant graphique. Quelle place pour la « substance » (substance de l'encre, opposée à la substance de l'air)? Quelle place pour la diachronie puisque une grammatologie à la Gelb n'est pas une histoire de l'écriture à la Février? Quelle

\footnotetext{
2 Avec la notion de « grammatisation » des langues, conçue comme le processus historique par lequel certaines se sont « outillées » (grammaires, dictionnaires, nomenclatures, paradigmes...), les historiens de la linguistique réactivent aujourd'hui à leur manière l'intérêt pour le "passage à l'écriture » de plusieurs point de vue : anthropologique, linguistique, psychologique (cf. Auroux, 1994).
} 
place pour le sujet? Quel degré d'extension admet le concept d'écriture pour conserver une compréhension minimale ? Quelle place pour le visible dans le lisible? Quelle place enfin pour les processus d'acquisition de l'écrit, étant entendu que la "genèse de l'écrit » quel que soit le sens individuel, social, ordinaire, esthétique... que l'on confère à ce terme, suppose l'adoption d'un point de vue spécifique ( $c f$. les travaux de Vygotski), une entrée nouvelle dans l'histoire de l'objectivation du "parler» ? C'est au confluent des ces perspectives que nous souhaitions revenir à Pau à propos de la question de «l'autonomie » de l'écriture.

Il nous semblait en effet que les vingt dernières années avaient en partie poursuivi, en partie renouvelé les approches de l'écriture léguées par les linguistes pour l'essentiel après la deuxième guerre mondiale. On pouvait alors dessiner de ce point de vue plusieurs directions.

- La première concernait l'intégration de la perspective sémiologique au cœur même de l'analyse linguistique, dans une sémiolinguistique qui resserre le point de vue sur la fonction linguistique du signe écrit. Pour cette perspective qui devait se rendre attentive aussi bien à la diversité des systèmes qu'à la diversité des signes à l'intérieur de chaque système (le "pluri-système » de N. Catach), l'autonomie de l'écriture n'est-elle que l'autonomie d'une zone bien réelle mais relativement marginale de certains systèmes d'écriture, ou un principe de description permettant d'accroitre la prise scientifique sur le matériau écrit ? Permettant de l'étendre par exemple aux dimensions de «l'espace graphique » dans son ensemble à partir d'une définition différente du "graphème » ('apport incontestable de notre ami J. Anis) ? Ici, le rapport privilégié de l'écriture à la langue est maintenu, mais c'est dans la manière même de concevoir cette relation que s'accomplissent sans doute les différenciations : l'autonomisme est-il une position méthodologique, une axiomatique de la description (plutôt référée à une linguistique générale proche de Hjelmslev) ? Le point de vue sémiolinguistique une position empirique, ontologique (plus proche d'un point de vue fonctionnaliste)?

- A l'autre extrémité du spectre, l'idée d'une sémiologie intégrationnelle et dynamique, celle de Roy Harris, refuse de renoncer à la richesse des statuts et des implications de l'écrit dans ses aspects à la fois cognitifs, sociaux, matériels, religieux, culturels au sens large... La relation de l'écriture à la langue perdait dans cette perspective son caractère fondateur et exclusif, pour s'intégrer dans une polyfonctionnalité, objet d'une véritable grammatologie réformatrice de la sémiologie saussurienne, différente et plus large que celle de Gelb, parce que reliée à une véritable antbropologie. Conçue contre une perspective phonographiste ou phonocentriste, cette perspective intégrationnelle est-elle pour autant autonomiste? Ou ne l'est-elle qu'avec quels types de réserves ? Dans quelle mesure ? Quel sens enfin doit-on conférer au terme «sémiologie » dans une entreprise qui reprend ce terme à Saussure - historiquement au moins - en contestant radicalement les principes sur lesquels repose la sémiologie saussurienne (sa définition du signe, en particulier)?

- D’un autre point de vue, (mais dans quelle proximité relative avec le précédent ?), pouvait-on considérer que le recentrage des recherches sur l'écriture autour de la notion d'inscription (Anne-Marie Christin) permettait de renouer le lien entre graphie et image, et de retrouver ainsi dans l'écriture même la puissance du visible et du sémantisme spatial refoulé par la culture occidentale? En forçant sans doute passablement les choses, ne pouvait-on se demander si l'insistance - très productive en effet parce que fortement «intégratrice», elle aussi - sur les notions de «support» et «d'écran», la 
conception de l'écriture comme transposition - et non reproduction mimétique - de la parole, n'obligeait pas à une révision de la notion d'autonomie? Bref, si le verbal et le visuel partagent une même caractéristique projective, leur indépendance mutuelle estelle autre chose que l'autre face de leur dépendance commune vis à vis du support spatial ?

- La psychologie des processus, quant à elle, avait investi avec tous ses moyens les procédures par lesquelles des sujets construisent leurs compétences de scripteurs/ lecteurs, dans une perspective génétique qui n'exclut pas toujours, mais complète parfois de manière problématique, celle de la tradition philologique, elle-même transformée par l'étude des manuscrits d'écrivains, des brouillons d'écoliers. A quel titre, selon quels axes, dans quelle mesure et avec quel sens du terme « autonomie » était-elle concernée par les débats sur l'autonomie de l'écriture?

- Enfin la littérature anglo-saxonne, ayant introduit, pour désigner l'ensemble des travaux, des disciplines et des perspectives impliquées dans toute recherche sur l'écriture (théorique, pédagogique, anthropologique, psychologique, esthétique...) le terme de literacy, dans quelle mesure la consécration de ce vocable marquait-elle la reconnaissance d'un champ de recherches à la fois unifié et autonome? Ne s'agissait-il que d'une version superficiellement modernisée de «l'horizon de projection » sémiologique des linguistiques structurales ? Quelle sorte d'autonomie s'y trouve implicitement revendiquée ?

\section{Le concept de littératie face au continent-écriture}

Il est donc clair qu'à Pau, dans l'optique de J. Anis et dans les discussions qui nous ont tous les trois réunis, l'attention était focalisée sur la connaissance des systèmes d'écriture et l'outillage requis pour leur analyse. Mais le concept de littératie ouvrait à partir de là deux voies complémentaires d'investigation : d'une part, il s'agissait de s'interroger sur l'usage et le sens conférés à chaque système d'écriture dans une société donnée, d'autre part de réfléchir au trajet qui mène de l'existence des systèmes d'écriture à la construction des discours.

S'il était légitime que les linguistes de l'écrit explorent la complexité et la spécificité de ces systèmes en analysant la mixité des principes qui y sont à l'œuvre et les questions de typologie qu'ils engagent, une attention était requise aux significations symboliques dont ces systèmes étaient investis et plus généralement à leurs relations avec les contextes de leur émergence et développement. Les travaux sur la littératie, l'œuvre de Jack Goody spécifiquement, montrent la relativité du « grand partage » entre oralité et écriture, entre cultures écrites et cultures orales et mettent en évidence un continuum où la mixité technique est inséparable des contextes anthropologiques. La multiplicité ouverte des pratiques inventoriées par les ethnologues révèle une diversification des fonctions de l'écriture régie par les environnements culturels et leur historicité : usage mnémonique ici, usage administratif ou économique là... Les effets " cognitifs » du stockage et de la communication écrite se manifestent au plan tant phylogénétique qu'ontogénétique: d'un côté, si l'oral n'interdit pas les activités de notation et de calcul, l'écrit démultiplie la puissance de ces opérations et la question est posée depuis longtemps de l'éventuelle précellence de tel système d'écriture (on connait le débat sur les vertus des systèmes alphabétiques d'écriture face à leurs concurrents idéographiques); d'un autre côté, la thèse de Vygotski sur la réorganisation totale des processus mentaux dès lors qu'un individu parvient à maitriser l'écriture consonne avec l'observation des 
activités sociales où se trouve engagée l'écriture et un système d'écriture spécifiquement, ouvrant un espace de discussion entre linguistes de l'écrit, psychologues de la cognition et ethnologues de l'écriture. D’où, par exemple, la nécessité de ne pas séparer les débats sur la lecture des conceptions générales de l'écriture et de l'acculturation à l'écrit.

S'il est un autre continuum que suggère le concept de littératie et que l'univers scolaire manifeste, à sa manière, de manière exemplaire, c'est celui qui conduit de l'apprentissage des lettres aux Belles-Lettres, pourrait-on dire, de l'entrée dans l'écrit à la culture littéraire ${ }^{3}$. L'argumentation de Goody sur ce qu'il est convenu d'appeler «littératures orales» et sur l'origine écrite de certaines œuvres réputées "orales» comme l'Iliade ouvre à une conception anthropologique de la littérature qui se préoccupe des problèmes de transmission, de mémoire, de mot à mot et de variantes, de répétition et d'originalité. Mais ce «continu » des systèmes aux discours n'empêche pas la pensée d'une différence entre textualité et scripturalité (ce que montraient certaines des communications à ce colloque de Pau, privilégiant l'un ou l'autre versant et que J. Anis avait approché sous le titre d'« espaces graphiques » dans la troisième partie de l'ouvrage de 1988). Il existe dans toutes les cultures des formes de réalités scripturales qui empruntent davantage à l'ordre paradigmatique qu'à celui de la syntagmatique textuelle, davantage à la verticalité et à la multidimensionnalité qu’à la linéarisation que se partagent la parole et la textualité. La liste comme modèle premier et canonique de l'organisation, du classement de données, fournit un modèle de mise en forme d'une économie scripturaire qui ne cessera de se complexifier permettant le développement des savoirs et la constitution de disciplines savantes difficilement appréhendables en dehors des capacités représentatives et symbolisatrices de la formalisation écrite.

S'il s'agit de «traduire» en quelque sorte littératie par la locution «culture de l'écrit », on voit bien l'immensité des intérêts de connaissance ici impliqués tout autant que la volonté de ne pas rendre incommensurables des recherches qui se déploient dans des horizons disciplinaires épistémologiquement et historiquement distincts. Il nous plait de conclure ces quelques lignes en pensant que Jacques Anis, linguiste et sémiologue de l'écrit, s'il ne plaçait pas ses travaux sous le vocable de littératie, a beaucoup apporté à la connaissance de la culture de l'écritt.

\footnotetext{
3 Jacques Anis avait en apparence fait un parcours inverse: de l'univers du poème à l'analyse technique des systèmes d'écriture, de la littérature aussi à l'informatique, mais toujours alliant la culture scientifique et technique et la culture des Humanités, suscitant le plus grand intérêt chez les spécialistes de la génétique textuelle par exemple.

${ }^{4}$ Nous avons été particulièrement heureux de travailler à nouveau et de reprendre les mêmes questions sous un autre angle avec Jacques Anis dans un séminaire de travail de Paris 3 dédié aux «abréviations graphiques» au début des années 2000 ( $f$. Andrieux-Reix et al., 2004). Dans ce numéro, Jacques examinait d'un point de vue sémiolinguistique la pratique des abréviations dans la communication électronique.
} 
Jean-Louis Chiss et Christian Puech

\section{RÉFÉRENCES BIBLIOGRAPHIQUES}

ANIS, J. avec CHISs, J.-L. et PuECH, C., 1988, L'écriture : théories et descriptions, Bruxelles, De Boeck.

ANDrieuX-Reix, N., Branca, S. et Puech C., 2004, Ecritures abrégées (notes, notules, messages, codes...), Paris, Ophrys Bibliothèque de « Faits de Langues ».

Auroux, S., 1994, La révolution technologique de la grammatisation, Liège, Mardaga.

Catach, N. (éd.), 1988, Pour une théorie de la langue écrite, Paris, CNRS.

Christin, A.-M., 1995, L'image écrite ou la déraison graphique, Paris, Flammarion.

FeVrier, J. G., 1984, Histoire de l'écriture, Paris, Payot.

GELB, I. J., 1973, Pour une théorie de l'écriture, Paris, Flammarion.

GoODY, J., 1979, La raison graphique, Paris, Éditions de Minuit.

HARris, R., 1993, La sémiologie de l'écriture, Paris, CNRS.

Hjelmslev, L., 1968, Prolégomènes à une théorie du langage, Paris, Éditions de Minuit.

Vygotski, L., 1985, Pensée et langage, Paris, Editions sociales.

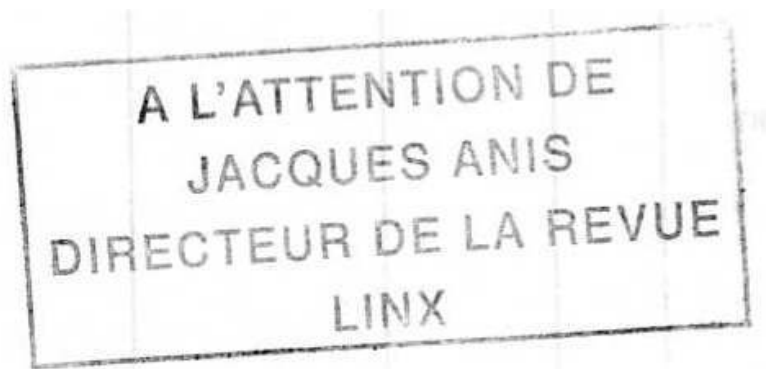

